\title{
Identificación de factores relacionados con la falta de participación en la detección de cáncer de mama por mastografía
}

\author{
Rodríguez-Nava VF*, Gómez-Cardona JP*, Rodríguez-Ramírez JM*, \\ Briones-Serna GC**, Guardado-Pizaña MG**, Pastrana-Aguilera AP, \\ Reyes-Acosta LA**, Rodríguez-Lemus F*
}

\begin{abstract}
Resumen
- Objetivo: Identificar los factores relacionados con la fal-

- ta de participación en la detección de cáncer de mama

- por mastografía. Material y métodos: Estudio descrip-

- tivo-trasversal, desarrollado mediante la aplicación de

- una encuesta a domicilio en mujeres residentes de la

- colonia Alianza Ferrocarrilera de la Ciudad de Aguasca-

- lientes de 45 a 65 años de edad, quienes participaron

- de manera voluntaria en la investigación en el mes de

- marzo de 201 I, mediante un cuestionario compuesto

- por diecinueve ítems, siete que describen las variables

- socio-demográficas y doce que identifican los posibles

- factores asociados con la no realización de la masto-

- grafía. Resultados: El 20.8\% de las mujeres encuesta-

- das coincidieron en que la primera razón por la que

- no acuden a realizarse la mastografía fue el miedo al

- resultado, seguida por la vergüenza y la falta de infor-

- mación. Conclusiones: Es necesario reforzar los méto-

- dos y estrategias de concientización de la población,

- con el objetivo de modificar los factores identificados, y

- con ello incrementar la participación de las mujeres en

- la prueba. LUXMÉDICA 7(20): II-19
\end{abstract}

\section{Abstract}

Objective: Identify factors related to lack of participation in the detection of breast cancer by mammography. Material and methods: A descriptive transversal study, developed through the application of a household survey in women living in the colony Alianza Ferrocarrilera in the city of Aguascalientes, 45 to 65 years of age, who volunteered to participate in research in the March 20 I I, a questionnaire with seven items that describe the socio-demographic variables and twelve to identify potential factors associated with not performing mammography. Results: $20.8 \%$ of women surveyed agreed that the first reason for not attending mammography performed the result was fear, followed by shame and lack of information. Conclusions:It is necessary to strengthen the methods and strategies for raising public awareness, with the aim of modifying the identified factors such as fear and shame to the result to show her breasts, and they result in increased participation of women in the test mammography. LUXMÉDICA 7(20): II-19

Keywords: Breast Cancer Factors, Mammography.

Palabras clave: Cáncer de mama, factores, mastografía.

* Profesores investigadores del Departamento de Enfermería de la Universidad Autónoma de Aguascalientes.

** Estudiantes de la licenciatura de Enfermería de la UAA

Fecha de recibido: 2 de diciembre del 2011

Fecha de aceptación: 23 de febrero del 2012

Correspondencia: MAE Víctor Federico Rodríguez Nava, Departamento de Enfermería del Centro de Ciencias de la Salud de la Universidad Autónoma de Aguascalientes. Avenida Universidad 940. CP 20131. Teléfono 9108437 correo electrónico victorrguez7@hotmail.com 


\section{Introducción}

El cáncer de mama es una de las principales causas de muerte en el mundo, según la Organización Mundial de la Salud (OMS), constituye una prioridad de salud no reconocida en países de ingreso medio, y se presenta una defunción cada dos horas producto de esta enfermedad ${ }^{1}$. En México a partir del año 2006, el cáncer de mama se posicionó como la primer causa de mortalidad por cáncer en mujeres de 30 a 54 años de edad; ${ }^{2}$ presentándose en mujeres menores de 54 años de edad en más del $50 \%$ de los casos $^{2}$. Cabe hacer mención, que la incidencia de cáncer de mama es directamente proporcional a la edad ${ }^{3,4}$ En Aguascalientes en el año 2005, la tasa de mortalidad por esta causa fue de 5.19 muertes por cada 100 mil habitantes en el intervalo de 15 a 64 años de edad, incrementándose en el año 2009 a 6.46, según cifras otorgadas por el Instituto de Servicios de Salud del Estado de Aguascalientes (ISSEA).

La mastografía es una técnica de eficacia comprobada, que permite detectar el cáncer mamario en etapa subclínica, tiempo en el cual las opciones terapéuticas y la posibilidad de curación son mayores ${ }^{5-7}$, situación que indudablemente, permite salvar muchas vidas. El fracaso en los programas para detectar este tipo de cáncer, es producto de la poca o nula participación en la prueba de mastografía 8 ; situación identificada en la población de estudio producto de un diagnóstico comunitario realizado durante el año 2009 por alumnos del Centro de Ciencias de la Salud de la Universidad Autónoma de Aguascalientes, circunstancia que generó la inquietud de realizar el presente estudio con la finalidad de identificar los factores por los cuales, las mujeres no participan en la prueba de tamizaje mamario.

| | | | | | | | | | | | | | | | | | | | | | | | | | | | | | | | | | | | | | | | | | | | | | | | | | | | | | | | | | | | | | | | | | | | | | | | | | | | | | | | | | | | | | | | | | | | | | | | | | | | | | | | | | | | | | | | | | | | | |

\section{Material y métodos}

Se realizó un estudio descriptivo-trasversal, mediante la aplicación de una entrevista a mujeres residentes en la colonia Alianza Ferrocarrilera de la Ciudad de Aguascalientes, las cuales al momento de realizar la entrevista se encontraban en un intervalo de 45 a 65 años de edad, quienes participaron de manera voluntaria en la investigación en el mes de marzo de 2011; excluyendo a quienes no desearan participar en la entrevista, no cumplieran con el rango de edad; y/o las encuestas no estuvieran contestadas en su totalidad.

La población de estudio fue de 110 mu- jeres, de entre las cuales se extrajo una muestra de 52 de ellas para la recolección de la información, utilizando el método de muestreo no probabilístico por conveniencia, tomando en consideración el cumplimiento de los criterios de selección.

El instrumento utilizado fue creado por los investigadores y revisado por un grupo de profesores expertos del departamento de enfermería de la Universidad Autónoma de Aguascalientes, mismo que está compuesto por diecinueve ítems, siete que describen las variables socio-demográficas y doce que identifican los posibles factores asociados a la falta de participación en la 
mastografía, en el cual se realizan preguntas cerradas de tipo nominal y ordinal.

Para el análisis de la información, se elaboró una base de datos en Epi-Info versión 3.3.2. Las variables fueron descritas me- diante frecuencias y porcentajes. Se utilizó la prueba estadística $X 2$ para relacionar las variables socio-demográficas con los factores por los que las mujeres no acuden a realizarse la mastografía.

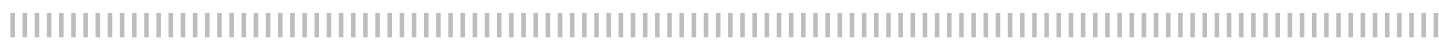

\section{Resultados}

Las 52 mujeres encuestadas, que residen en la colonia Alianza Ferrocarrilera, presenta- ron una edad promedio de $53.79 \pm 5.6$ años con una moda de 50 . El resto de las características se describen en las tablas 1 y 2.

\section{Tabla l}

\section{Descripción de variables socio-demográficas}

\begin{tabular}{|c|c|c|}
\hline VARIABLES & FRECUENCIA & PORCENTAJE \\
\hline \multicolumn{3}{|l|}{ Edad } \\
\hline 45-55 años & 31 & 59.6 \\
\hline 56-65 años & 21 & 40.4 \\
\hline \multicolumn{3}{|l|}{ Religión } \\
\hline Católico & 46 & 86.8 \\
\hline Cristiana & 3 & 5.7 \\
\hline Testigo de Jehová & 3 & 5.7 \\
\hline \multicolumn{3}{|l|}{ Ocupación } \\
\hline Hogar & 42 & 79.2 \\
\hline Empleada & 6 & 11.3 \\
\hline Desempleada & 2 & 3.8 \\
\hline \multicolumn{3}{|l|}{ Estado civil } \\
\hline Casada & 36 & 67.9 \\
\hline Soltera & 7 & 13.2 \\
\hline Viuda & 6 & 11.3 \\
\hline \multicolumn{3}{|c|}{ Institución de afiliación } \\
\hline IMSS & 20 & 37.7 \\
\hline Seguro popular & 15 & 28.3 \\
\hline \multicolumn{3}{|l|}{ Escolaridad } \\
\hline Primaria & 36 & 67.9 \\
\hline Secundaria & 7 & 13.2 \\
\hline \multicolumn{3}{|l|}{ Ingreso mensual } \\
\hline$\leq \$ 2000$ & 24 & 45.3 \\
\hline$\$ 2001$ a $\$ 3000$ & 18 & 34.0 \\
\hline \multicolumn{3}{|l|}{ No. de hijos } \\
\hline 0-4 hijos & 26 & 50.0 \\
\hline 5-9 hijos & 23 & 44.2 \\
\hline
\end{tabular}

Fuente: Entrevista a mujeres del Fraccionamiento Alianza ferrocarrilera, Marzo 2011 


\section{Tabla 2}

\section{Descripción de variables relacionadas con la falta de participación en la mastografía}

\begin{tabular}{|c|c|c|}
\hline & FRECUENCIA & PORCENTAJE \\
\hline \multicolumn{3}{|c|}{ Que es la mastografía } \\
\hline $\mathrm{Si}$ & 26 & 49.1 \\
\hline No & 20 & 37.7 \\
\hline Poco & 6 & 11.3 \\
\hline \multicolumn{3}{|c|}{ Dónde realizarse el estudio de la mastografía } \\
\hline $\mathrm{Si}$ & 29 & 54.7 \\
\hline No & 23 & 43.4 \\
\hline \multicolumn{3}{|c|}{ Lugar donde se realiza la mastografía se encuentra: } \\
\hline Cerca & 31 & 58.5 \\
\hline Lejos & 15 & 28.3 \\
\hline Muy lejos & 6 & 11.3 \\
\hline \multicolumn{3}{|c|}{ Importancia de la mastografía } \\
\hline Mucha & 46 & 86.8 \\
\hline Poca & 3 & 5.7 \\
\hline Me es indiferente & 2 & 3.8 \\
\hline Ninguna & 1 & 1.9 \\
\hline \multicolumn{3}{|c|}{ Tiene miedo a conocer el posible resultado } \\
\hline $\mathrm{Si}$ & 27 & 50.9 \\
\hline No & 25 & 47.2 \\
\hline \multicolumn{3}{|c|}{ Importancia de mostrar los pechos al personal de salud } \\
\hline Mucho & 19 & 35.8 \\
\hline Regular & 10 & 18.9 \\
\hline Poco & 6 & 11.3 \\
\hline Nada & 17 & 32.1 \\
\hline
\end{tabular}

Fuente: Entrevista a mujeres del Fraccionamiento Alianza ferrocarrilera, Marzo 2011

El $20.8 \%$ de las mujeres encuestadas coincidieron en que la primera razón por la que no acuden a realizarse la mastografía fue el miedo al resultado, seguida por la vergüenza y la falta de información.
La relación entre los principales factores identificados con la no participación en la mastografía y las variables socio-demográficas con mayor relevancia encontrada se describe en la tabla 3. 


\section{Tabla 3}

Descripción de relación entre variables socio-demográficas y las tres primeras causas identificadas en la omisión de la realización de la mastografía.

\begin{tabular}{|c|c|c|c|c|}
\hline \multicolumn{5}{|c|}{ Primera causa } \\
\hline & Miedo al resultado & $\begin{array}{l}\text { Pena de mostrar } \\
\text { los pechos }\end{array}$ & Falta de información & Otros \\
\hline \multicolumn{5}{|l|}{ Escolaridad } \\
\hline Primaria & 14 & 7 & 1 & 14 \\
\hline Secundaria & 3 & 1 & 1 & 4 \\
\hline Bachillerato & 0 & 0 & 0 & 1 \\
\hline Nivel Superior & 0 & 0 & 0 & 1 \\
\hline Ninguno & 1 & 1 & 0 & 3 \\
\hline Total & 18 & 9 & 2 & 23 \\
\hline \multicolumn{5}{|l|}{ Ingreso mensual } \\
\hline Menos de $\$ 2000$ & 4 & 5 & 1 & 11 \\
\hline$\$ 2001-\$ 3000$ & 8 & 0 & 1 & 7 \\
\hline$\$ 3001-\$ 5000$ & 1 & 1 & 0 & 4 \\
\hline Más de $\$ 5000$ & 0 & 1 & 0 & 0 \\
\hline Total & 13 & 7 & 2 & 22 \\
\hline \multicolumn{5}{|c|}{ Segunda causa } \\
\hline & $\begin{array}{l}\text { Pena a mostrar } \\
\text { los pechos }\end{array}$ & No tengo dinero & Miedo al resultado & Otros \\
\hline \multicolumn{5}{|l|}{ Escolaridad } \\
\hline Primaria & 6 & 5 & 5 & 20 \\
\hline Secundaria & 0 & 2 & 1 & 6 \\
\hline Bachillerato & 0 & 1 & 0 & 0 \\
\hline Nivel Superior & 1 & 0 & 0 & 0 \\
\hline Ninguno & 1 & 0 & 1 & 3 \\
\hline Total & 8 & 8 & 7 & 29 \\
\hline \multicolumn{5}{|l|}{ Ingreso mensual } \\
\hline Menos de $\$ 2000$ & 2 & 5 & 2 & 15 \\
\hline$\$ 2001-\$ 3000$ & 4 & 3 & 1 & 12 \\
\hline$\$ 3001-\$ 5000$ & 2 & 0 & 3 & 2 \\
\hline Más de $\$ 5000$ & 0 & 0 & 1 & 0 \\
\hline Total & 8 & 8 & 7 & 29 \\
\hline \multicolumn{5}{|c|}{ Tercera causa } \\
\hline & Falta de información & $\begin{array}{c}\text { Dolor } \\
\text { al procedimiento }\end{array}$ & $\begin{array}{l}\text { Pena de mostrar } \\
\text { los pechos }\end{array}$ & Otros \\
\hline \multicolumn{5}{|l|}{ Escolaridad } \\
\hline Primaria & 5 & 6 & 5 & 20 \\
\hline Secundaria & 3 & 2 & 1 & 3 \\
\hline Bachillerato & 0 & 0 & 0 & 1 \\
\hline Nivel Superior & 0 & 0 & 1 & 0 \\
\hline Ninguno & 1 & 0 & 0 & 4 \\
\hline Total & 9 & 8 & 7 & 28 \\
\hline \multicolumn{5}{|l|}{ Ingreso mensual } \\
\hline Menos de $\$ 2000$ & 3 & 4 & 1 & 16 \\
\hline$\$ 2001-\$ 3000$ & 6 & 2 & 3 & 9 \\
\hline$\$ 3001-\$ 5000$ & 0 & 0 & 3 & 2 \\
\hline Más de $\$ 5000$ & 0 & 0 & 0 & 1 \\
\hline Total & 9 & 8 & 7 & 28 \\
\hline
\end{tabular}

Fuente: Entrevista a mujeres del Fraccionamiento Alianza ferrocarrilera, Marzo 2011 
Al relacionar los principales factores identificados como la primera razón por la que no se realizan la mastografía, se mostró una distribución similar para la variable escolaridad y el ingreso mensual, no encontrándose diferencias estadísticamente significativas.

\section{||||||||||||||||||||||||||||||||||||||||||||||||||||||||||||||||||||||||||||||||||||||||||||||}

\section{Discusión}

El miedo al resultado y la vergüenza de mostrar los pechos, al personal de salud, son los dos primeros factores por los que las encuestadas no acuden a realizarse la mastografía, datos que coinciden con los descritos en un estudio realizado por el IMSS (2007) ${ }^{9}$.

La "falta de información", ocupó el tercer lugar entre las causas de no participación en la prueba de detección, factor identificado en un estudio realizado en los estados de Oaxaca y el Distrito federal, donde refieren que a mayor conocimiento sobre la enfermedad, su diagnóstico y pronóstico, mayor participación en la mastografía. ${ }^{10}$ En relación al ingreso mensual, se encontró que el ingreso bajo de las encuestadas fue un motivo para la no asistencia a los centros donde se realiza la mastografía, resultado que es similar al encontrado por otros autores, Sosa (2009) ${ }^{11}$ y Anderson $(2007)^{12}$, ambos refieren que la baja participación de las mujeres en el examen de la mastografía se debe a la pobreza.

Así mismo, tener un nivel de escolaridad bajo, se considera otra razón para que las mujeres no se realicen la mastografía, coincidiendo con los resultados arrojados en un estudio realizado en Valencia (España). ${ }^{8}$

\section{Conclusiones}

La falta de información retrasa sin lugar a dudas la asistencia de las mujeres a la realización de la mastografía ${ }^{13}$, ya que no lo consideran importante. ${ }^{14}$ Es por ello, que se considera necesario reforzar los métodos y estrategias de concientización de la población en general y a los proveedores de servicios de salud ${ }^{15}$, con el objetivo de que tengan una mayor información sobre el cáncer de mama, los métodos diagnósticos preventivos de la enfermedad y la importancia de su realización ${ }^{16}$, lo que derivará en la modificación de los factores tales como el miedo al resultado y vergüenza a mostrar los pechos, incrementándose así, la participación de las mujeres en la prueba de la mastografía.

\section{Bibliografía}

1. Knaul F.M, Nigenda G, Lozano R, Arreola-Ornelas R, Langer A, Frenk J. Cáncer de mama en México: una prioridad apremiante. Salud Pública de México [en línea]. 2009. URL disponible en: http://www.scielo. org.mx/scielo.php?pid=S0036-363420090008000 26\&script $=$ sci_arttext
2. Martínez-Cruz G, Juárez-Ramiro A, Pichardo-Cuevas $M$, Martín-Ordoñez A. Cáncer de mama en mujeres menores de 40 años. Una evolución desfavorable cada vez más frecuente en la práctica clínica. GAMO [en línea]. 2010. URL disponible en: http://www. elsevier.es/sites/default/files/elsevier/pdf/305/ 305v09n06a90000429pdf001.pdf 
3. Ester Brandan M Villaseñor Navarro $Y$. Detección del Cáncer de Mama: Estado de la Mamografía en México. Cancerología [en línea]. 2006. URL disponible en: http://www.incan.org.mx/revistaincan/elementos/documentosPortada/1172289111.pdf

4. Pilar Rubio J. P. Tesis doctoral. Análisis del programa de detección precoz del cáncer de mama en Jaén y del carcinoma de intervalo en Andalucía [en línea]. 2003. URL disponible en: http://www-rayos.medicina.uma.es/Rmf/Tesis/Tesis_doctoral_Jose_P_Rubio. pdf

5. Vázquez A. Valor de la mamografía en la detección temprana del cáncer mamario. Biomedicina [en línea]. 2010. URL disponible en: http://www.medicare. com.uy/files/articulos-cientificos/valor_de_la_mamografia_en_la_deteccion_temprana_del_cancer. pdf

6. Asua Batarrita J. Mamografía y detección precoz del cáncer de mama. Revista Española de Salud Publica [en línea]. 2005. URL disponible en: http://scielo. isciii.es/scielo.php?pid=S1135-5727200500050000 $1 \&$ script $=$ sci_arttext

7. Peralta A, Lazcano Á, Reyes Y, Redondo F, Marti Julia, Ríos N. Reporte de lesiones no palpables diagnosticadas por mastografía en el Hospital General de México. Anales de Radiología México [en línea]. 2008. URL disponible en: http://www.artemisaenlinea.org.mx/acervo/pdf/anales_radiologia_mexico/ $5 \%$ 20reporte $\%$ 20de \% 20lesiones.pdf

8. Alcaraza $M$, Lluch $A_{1}$. Miranda J, Pereiro I, Salas M.D. Estudio de la no participación en el programa de prevención de cáncer de mama en la ciudad de Valencia. Gac Sanit [en línea]. 2002. URL disponible en: http://scielo.isciii.es/scielo.php?pid=S021391112002000300006\&script=sci_arttext

9. Torres-Arreola L.P, Vladislavovna Doubova S. Cáncer de mama. Detección oportuna en el primer nivel de atención. Revista médica del Instituto Mexicano del Seguro Social [en línea]. 2007. URL disponible en: http://www.medigraphic.com/pdfs/imss/im2007/im072i.pdf

10. Nigenda G, Caballero M, González-Robledo L. M. Barreras de acceso al diagnóstico temprano del cáncer de mama en el Distrito Federal y en Oaxaca. Salud Pública de México [en línea]. 2009. URL disponible en: http://www.scielo.org.mx/scielo. php?pid=S0036- 36342009000800016\&script $=$ sci _arttext

11. Sosa-Rubí S.G, Walker D, Serván E. Práctica de mastografías y pruebas de Papanicolaou entre mujeres de áreas rurales de México. Salud Pública de México [en línea]. 2009. URL disponible en: http://www.scielo. org. $\mathrm{mx} /$ scielo.php? script =sci arttext\&pid $=$ S003636342009000800014

12. Anderson B. O, Yip C.H, Ramsey S, Bengoa R, Braun $S$, Fitch M. El Cáncer de mama en los países con recursos limitados: Sistemas de atención de salud y políticas públicas. The Breast Journal [en línea]. 2007.
URL disponible en:http://www.paho.org/spanish/ $\mathrm{ad} / \mathrm{dpc} / \mathrm{nc} / \mathrm{pcc}$-bc-bhgi-normas-int-5.pdf

13. Wiesner C. Determinantes psicológicos, clínicos y sociales del diagnóstico temprano del cáncer de mama en Bogotá, Colombia. Revista Colombiana de Cancerologia [en línea]. 2007. URL disponible en: http:// www.cancer.gov.co/documentos/RevistaCC2007\% 20Vol\% 2011\% 281\% 29/rccv11n1a03.pdf

14. Serral Cano G, Puigpinós i Riera R, Robles Garrido I, Pons-Vigués $M$, Borrell $C$. Satisfacción y expectativas de las mujeres participantes y no participantes en el programa de detección precoz de cáncer de mama de Barcelona tras 10 años de funcionamiento. Revista española de Salud Publica [en línea]. 2010. URL disponible en: http://scielo.isciii.es/scielo. php? pid $=$ S1135-57272010000600004\&script $=$ sci arttext

15. Knaul F. M, López Carrillo L, Lazcano Ponce $E$, Gómez Dantés H, Romieu I, Torres G. Cáncer de mama: un reto para la sociedad y los sistemas de salud. Salud Pública de México [en línea]. 2009. URL disponible en: http://www.scielo.org.mx/scielo. php?pid $=$ S0036-36342009000800002\&script $=$ sci arttext

16. Andreu Vallio $Y$, Galdon Garrido M. J, Dura Ferrandis E, Carretero Gomez S, Tuells Hernandez J. Edad, creencias de salud y asistencia a un programa de cribado mamografico en la comunidad Valenciana. Revista española de Salud Publica [en línea]. 2004. URL disponible en: http://scielo.isciii.es/scielo. php?pid $=$ S1135 $-57272004000100007 \&$ script $=$ sci arttext

17. López R. O. La epidemia de cáncer de mama en México. ¿Consecuencia de la transición demográfica? Centro de Investigación en Salud Poblacional, Instituto Nacional de Salud Pública, México 1997.

18. Aspectos psicológicos del cáncer de mama. Disponible en: http://enfermeriacancerdemama.blogspot. com/2008/02/aspectos-psicolgicos-del-cncer-demama.html

19. Ruiz M. A. Garde S. Intervención psicológica en pacientes con cáncer de mama. Departamento de Psicología de la Personalidad, Evaluación y Tratamiento Psicológicos. Facultad de Psicología. UNED. Disponible en: http://www.cfnavarra.es/salud/anales/textos/vol21/suple3/pdf/15inte.pdf

20. Aseguran que mujeres rechazan la mastografía por "temor" a la verdad México (Notimex). Disponible en: http://www.elporvenir.com.mx/notas. asp?nota_id $=136443$ 


\section{INSTRUMENTO \\ FACTORES ASOCIADOS ANTE LA OMISIÓN DE LA MASTOGRAFÍA}

\section{Datos sociodemográficos}

INSTRUCCIONES: Lea cuidadosamente y conteste lo que continuación se le pide. (Subraye o marque con una " $X$ ")

NOTA: La información proporcionada será meramente confidencial y usada para fines de investigación.

1. Edad:

2. Religión:

Católica Ocristiana OTestigo de Jehová $O$ Otro (especifique)

3. Ocupación:
Hogar
Obrera
Empleada
Desempleada

Otro (especifique)

4. Estado civil:
$\bigcirc$ Casada
$\bigcirc$ Soltera
$\bigcirc$ Divorciada
Viuda

5. Afiliada a:
OIMSS
Cissste
Seguro Popular
Oportunidades

6. Escolaridad:

$\bigcirc$ Primaria

$\bigcirc$ Secundaria

$\bigcirc$ Bachillerato

Nivel superior

7. Ingreso Mensuales:

$\bigcirc$ Menos de $\$ 2,000$

De $\$ 2,001$ a 3,000

De $\$ 3,001$ a 5,000

Mayor a $\$ 5,000$

Factores relacionados con la falta de participación en la prueba de Mastografía

1. ¿Número de hijos?

2. ¿Sabe usted qué es la mastografía?<smiles>[Si]C1CCCCC1</smiles><smiles>[O+]=[N+]=[Nb]</smiles><smiles>O=[Po](O)C1CCCCC1</smiles>

3. ¿Sabe dónde realizarse el estudio de la mastografía?<smiles>CCCC[SiH2]</smiles><smiles>[Nb]=[No]</smiles>

4. ¿Considera que el lugar donde se realiza la mastografía se encuentra:
Cerca
Lejos
Muy lejos 
5. ¿Cuál es su medio de transporte?
Automóvil propio
Transporte Público Bicicleta
Ninguno

6. ¿Qué importancia tiene para usted la detección oportuna de cáncer de mama por medio de mastografía?
Mucha
Poca
$\bigcirc$ Me es indiferente

7. ¿Tiene miedo a realizarse la mastografía?<smiles>[N-]=[N+]1CCCCCC1</smiles>

8. ¿Tiene miedo a conocer el posible resultado?
$\bigcirc \mathrm{si}$
$\bigcirc$ No

9. ¿Qué importancia tiene para usted mostrar los pechos al personal de salud?
$\bigcirc$ Mucho
$\bigcirc$ Regular
Poco
$\bigcirc$ Nada

10. ¿Algún miembro de su familia se opone a que se realice la mastografía?
Esposo y/o cónyuge
$\bigcirc$ Hijos
$\bigcirc$ Ninguno

11. ¿Alguna de sus prácticas religiosas o culturales se oponen con la realización de la mastografía?

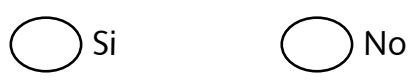

INSTRUCCIONES: Marque las 5 causas por las que no acude a realizarse la prueba de Mastografía, numerándolas de menor a mayor de acuerdo al orden de importancia que tiene para usted;. (El número 1 corresponde a MAS importante y 5 como MENOS IMPORTANTE)

12. ¿Cuál es la principal causa por la cual no se realiza la mastografía?

Miedo al resultado

Vergüenza a mostrar su cuerpo

Dolor al procedimiento

( )

( )

Tradiciones/ costumbres/religión me lo impiden ( )

No cuento con servicio médico ( )

Falta de información ( )

No tengo tiempo ( )

La distancia de la unidad Médica ( )

Desaprobación de mi esposo ( )

No tengo dinero ( )

Me da flojera asisti ( )

A mí no me va a pasar $\quad($ )

Nadie me dijo que tengo que hacérmelo $($ ) 